\title{
Rursus
}

Russus

Poiétique, réception et réécriture des textes antiques

$6 \mid 2011$

Relire, récrire, prolonger. Adaptations gréco-latines

\section{L'extension du vocabulaire de la beauté dans le De amore, IV, de Marsile Ficin}

Evrard Delbey

\section{OpenEdition}

Journals

Édition électronique

URL : http://journals.openedition.org/rursus/500

DOI : 10.4000/rursus.500

ISSN : 1951-669X

Éditeur

Université Nice-Sophia Antipolis

Référence électronique

Evrard Delbey, «L'extension du vocabulaire de la beauté dans le De amore, IV, de Marsile Ficin », Rursus [En ligne], 6 | 2011, mis en ligne le 28 janvier 2011, consulté le 19 avril 2019. URL : http:// journals.openedition.org/rursus/500 ; DOI : 10.4000/rursus.500

Ce document a été généré automatiquement le 19 avril 2019

Rursus 


\title{
L'extension du vocabulaire de la beauté dans le De amore, IV, de Marsile Ficin
}

\author{
Evrard Delbey
}

- Dans Le Banquet de Platon, Aristophane affirme que dans le monde mythique d'autrefois il existait trois types d'êtres humains. Tu connais cette histoire?

- Non.

- Autrefois, les êtres humains ne naissaient pas homme ou femme, mais homme/homme, homme/ femme ou femme/femme. Autrement dit, il fallait réunir deux personnes d'aujourd'hui pour en faire une seule. Tout le monde était satisfait comme ça, et la vie se déroulait paisiblement. Mais Dieu a pris une épée et a coupé tous les êtres en deux bien nettement, par le milieu. Résultat : il y a eu des hommes et des femmes, et les gens se sont mis à courir dans tous les sens toute leur vie à la recherche de leur moitié perdue.

- Pourquoi Dieu a-t-Il fait ça?

- Couper les gens en deux? Je n'en sais rien, moi.

Ce que fait Dieu est généralement assez incompréhensible. Il se met facilement en colère et puis, comment dire, Il a une tendance à l'idéalisme. J'imagine que c'était une punition. Comme dans la Bible, quand Il a chassé Adam et Eve du paradis. - Le péché originel, dis-je.

- Oui, le péché originel. (Il tient son crayon en équilibre entre l'index et le majeur et le fait 
osciller lentement.) En fait, je voulais dire que c'est
difficile pour un humain de vivre seul.
Je retourne dans la salle de lecture lire la suite de
l'histoire d'Abou-Assan le bouffon. Mais j'ai du mal
à me concentrer. Homme/homme, homme/femme
ou femme/femme?
(H. Murakami, Kafka sur le rivage, p. 52-53)

\section{Introduction}

Le discours d'Aristophane, tel que nous le livre Platon, a suscité en effet de nombreux commentaires. L'un des plus fameux est celui que nous lisons au Livre IV du De amore composé par Marsile Ficin. Cet ouvrage dédié à Jean Cavalcanti, l'ami de Ficin, rédigé en 1469, est de tous les Commentaires écrits par Ficin le seul qui soit achevé. Intitulé Commentarium in Conuiuium Platonis, il contribue à l'élaboration d'un système de pensée dont le néoplatonisme se veut lumineux, exaltant la centralité de l'individu et la rédemption de son âme immortelle par la beauté et l'amour. De fait, Ficin travaille à l'harmonisation du monothéisme chrétien avec la pensée platonicienne et le De amore constitue ainsi le prélude sublime de la Théologie platonicienne.

Notre propos est de montrer comment Ficin réévalue la beauté physique en empruntant à Platon l'idée que l'amour profane d'un beau corps est la réminiscence de l'amour sacré pour Dieu (voir l'éloge de l'éraste dans le Phèdre, 250e-252c). Relisant Platon à la lumière du pseudo-Denys l'Aréopagite, Ficin compose donc un seul et même septuple discours, à partir du Banquet, sur l'amour ramenant l'âme au sein de l'Un divin. Comme le note P. Laurens, dont nous suivons l'édition du texte de Ficin parue aux Belles Lettres en 2002 :

«Une différence essentielle, en effet, avec le Banquet platonicien où, on l'a dit, les intervenants ne se faisaient pas faute de mettre en cause les thèses exposées avant eux, est le consensus qui, chez Ficin, s'établit tout naturellement entre les participants, amis d'une même doctrine. (...) chacun, en amorçant son propre discours, prendra soin de valider les points déjà mis en lumière par ses prédécesseurs, pour s'appuyer sur eux et aller plus loin dans l'exposé de la thèse. » (Laurens 2002: XXXVIII)

Pour notre part, nous avons choisi d'étudier l'extension du vocabulaire de la beauté dans le commentaire, fait par le poète platonicien Cristoforo Landino, du discours d'Aristophane qui, dans le texte de Platon, n'utilise pas le concept de beauté précisément. Que révèle cette expansion lexicale? Les quatre premiers discours-commentaires -celui de Landino est précisément le quatrième- "servent à dessiner l'image de l'univers théocentrique et à imposer l'idée dionysienne de la conuersio ou retour circulaire a bono in bonum, en passant par la beauté, puis, une fois marquée la place de l'âme au point d'équilibre de la hiérarchie, à concevoir la possibilité pour elle de monter ou de descendre selon qu'elle suit l'une ou l'autre des deux Aphrodites.» (Laurens 2002 : XXXIX). Dans l'esprit de Ficin, le Banquet est étroitement lié au Phèdre:

«Or le Phèdre n'est pas seulement l'œuvre de Platon qui expose complètement la théorie des quatre fureurs, dont une, la fureur amoureuse, est présente dans le Banquet et une autre, la fureur poétique, dans l'Ion : il est aussi, selon une tradition erronée mais admise, le premier en date et en ce sens séminal, le plus génial aussi $\mathrm{du}$ canon platonicien, étant dans le cadre exceptionnel de son paysage idyllique au bord de l'Illissos, le produit de la jeunesse, de l'amour et de la poésie. » (Laurens 2002 : LIX). 
D'où de notables différences dans le mode de réception du discours d'Aristophane entre Ficin et Platon.

\section{Le discours d'Aristophane dans Le Banquet de Platon}

Dans le Banquet de Platon (189a - 193d) en effet, Aristophane fait partie de ces rhéteurs ou sophistes qui expriment des points de vue étrangers à la philosophie. Socrate oppose une fin de non-recevoir à l'égard de tout ce qui a été dit avant lui et affirme énergiquement la nécessité d'employer désormais une méthode radicalement différente pour définir l'Amour. Léon Robin pense que Platon "a voulu traiter Aristophane en adversaire.» (Robin1930 : LVII). Il le représente saisi d'un violent hoquet qui le rend incapable de parler après Pausanias et en fait le personnage ridicule d'un intermède burlesque (185ce), ivrogne de surcroît (176b). Platon n'a pas oublié le poète comique qui, en calomniant Socrate, a contribué à exciter les haines qui ont perdu ce dernier. Aristophane, remis de son hoquet, grâce aux prescriptions amusées d'Eryximaque, médecin de son état, parlera finalement après celui-ci. Le discours d'Alcibiade sera une réponse à la caricature de Socrate perdu dans les nuées de ses méprisables sophismes, tel qu'Aristophane l'avait représenté, et Platon fera entrer Alcibiade au moment où, seul parmi tous les assistants, Aristophane veut élever une protestation contre le discours de Socrate (212c). Et Robin, non sans vraisemblance, considère que Platon, par le discours qu'il fait tenir au poète, s'amuse à l'imiter : c'est « le scénario d'une comédie féerique dans le genre de ce que sont les Oiseaux. On s'imagine en effet sans peine un chœur bouffon d'hommes d'une seule pièce et tout en boule, avec leurs huit membres, leurs deux visages, leurs attributs sexuels en double et, dans le cas des androgynes, contraires sur chaque face, faisant enfin la roue sur scène (189d sqq.) : chœur étrange et bien propre à exciter la gaité populaire! Voici maintenant, au milieu d'eux, les protagonistes hardis d'une entreprise contre l'olympe (190bc). Bientôt, nous assisterons au conseil des dieux menacés; nous entendrons le discours de Zeus (190c-e); nous serons témoins de toute cette chirurgie et prothèse apollinienne qui, selon les modifications qu'exige le plan d'abord arrêté, doivent peu à peu donner naissance à l'humanité actuelle (190 e sq., 191a-c). On croit voir, maintenant dédoublés, ces hommes massifs du début ; on devine quelles expressions lyriques seraient données à l'aspiration de chaque moitié vers la moitié qui lui correspond, au désespoir de la recherche infructueuse, à la joie, trop rare, de s'être enfin réunie à la moitié qui la complète et avec laquelle elle reconstituera son unité primitive (191ab, d sqq., 193b-c). A présent, c'est l'apparition d'Héphaïstos, armé de ses outils de forgeron; la scène est ébauchée : il offre aux moitiés qui se sont ainsi retrouvées de les souder définitivement l'une à l'autre (192d sqq.). Enfin, une conclusion morale : nous sommes des êtres déchus, dont l'impiété a causé la déchéance ; l'amour est le seul remède à notre misère, l'unique moyen de notre salut par le retour à l'état de choses d'autrefois (189d, 191d, 193d) ; mais nous tomberons plus bas encore si nous revenons aux fautes qui nous ont perdus (190d, 193ab). - Bref nous trouvons ici les caractères les plus essentiels de la comédie aristophanesque : une thèse et une affabulation dont elle se revêt, mélange étourdissant de bouffonnerie effrénée et d'admirable poésie (...)»(p. LIX-LX). Car Robin considère aussi que Platon a voulu que la thèse d'Aristophane, sans être soutenue par la philosophie, fût la plus profonde de toutes celles qu'expose la première partie du Banquet : «(...) c'est ce qu'on peut appeler la théorie de l'âme-sœur, et Aristophane est en droit de dire que, par elle, il a rompu (...) avec le point de vue même de Pausanias et d'Eryximaque. Il 
abandonne la distinction des deux Amours : pour lui l'amour est un dans son essence, et sa fonction est de recréer l'unité; c'est d'autre part à une sorte de mystère qu'il se propose d'initier ceux qui l'écoutent (189d), car l'amour contient tout le mystère de notre destinée. » (p. LX). A l'égard de l'amour masculin, enfin, l'attitude d'Aristophane semble contrastée : il en parle en termes flatteurs (191 e-192b), mais à deux reprises (192bc, 193c), il insiste sur l'idée que sa théorie concerne aussi bien les femmes.

Que devient alors, chez Ficin, cet exposé mi-parodique mi-sérieux sur l'amour, union des amants en une seule âme? Pour le comprendre, il convient de rappeler les acquis des trois premières interventions.

\section{Les trois premiers commentaires dans le De amore}

7 C'est Giovanni Cavalcanti, l'ami de Ficin, qui est chargé des trois premiers commentaires : celui des discours de Phèdre, de Pausanias et d'Eryximaque. Comment utilise-t-il le vocabulaire de la beauté ? C'est le mot pulchritudo qui traduit le sujet du Phèdre (I.2) dans le premier commentaire. L'Amour succède immédiatement au Chaos, il précède le monde et les dieux ; c'est lui qui entraîne vers la beauté :

Le charme de ce monde ou ornement, c'est la beauté < pulchritudo >, vers laquelle l'Amour, dès qu'il est né entraîne et conduit l'Intelligence, cette même Intelligence d'abord difforme et sans beauté et qui acquiert ensuite forme et beauté < mentem ante deformem ad mentem eamdem deinde formosam >. Aussi est-ce la vocation même de l'amour d'entrâner vers et d'unir belle forme et difformité < Ideo amoris conditio est, ut ad pulchritudinem rapiat ac deformem formoso coniungat >. (De amore, I.3)

8 Grâce à l'Amour, l'Intelligence se tourne vers Dieu, comme l'œil vers la lumière. Amour format informia, donne forme à l'informe ; ainsi pulchritudo et forma se combinent pour que nous évitions le laid, autrement dit le mal et le déshonnête, en enseignant le pudor :

(...) le mal pour l'homme se confond avec le déshonnête (...) < Mala hominis et turpia eadem sunt $>($...) la pudeur proscrit les actes déshonnêtes $(. .)<.(.$.$) a turpibus pudor$ absterret >. (I.4)

9 Après avoir défini l'Amour comme étant désir de beauté (I.4 : pulchritudinis desiderium), Cavalcanti définit alors de manière exhaustive ce qu'est la Beauté ; elle est une grâce ( gratia), un accord (concinnitas) satisfait exclusivement par l'esprit, les yeux et les oreilles :

En effet dans les âmes la grâce résulte de l'accord entre plusieurs vertus; elle naît dans les corps de l'harmonie de plusieurs couleurs et lignes; et enfin, dans les sons, de la consonance parfaite de plusieurs sons. Par suite il y a une triple beauté : celle des âmes, des corps, des sons. Celle des âmes est perçue par l'esprit, celle des corps par les yeux, celle des sons par les oreilles exclusivement (I.4)

10 Ni l'odorat, ni le goût, ni le toucher ne constituent la beauté humaine : ils appartiennent au désir brut (appetitio, libido cohitus, libido coeundi, rabies uenerea, lasciuia) qui est concupiscence, laideur (deformitas). D'où le lien entre l'Amour, la beauté et la décence ( decora). Ainsi le premier commentaire établit la connexion suivante: Amor > pulchritudo > forma $>$ animi decor.

11 Le commentaire du discours de Pausanias revient sur la définition de l'Amour comme Beauté divine. Dieu a engendré l'Amour pour que l'homme soit attiré par sa Beauté essentielle. Bon, lorsqu'il crée, Dieu est Beau, lorsqu'il attire à lui : la pulchritudo dérive de cette divine bonitas (II.1). Dans le titre même du chapitre 3, nous pouvons lire la formule suivante : «La Beauté est splendeur de la Bonté divine» ( Pulchritudo est splendor diuine 
bonitatis). Le rayon de la Beauté modèle les formes dans la Matière (II.3), mais si nous avons l'habitude de juger du divin d'après ce que nous voyons de supérieur dans les corps, il est vrai que les formes corporelles ne montrent pas suffisamment les choses divines; « (...) les formes des corps semblent plutôt des ombres que des choses véritables. » (II.4).

Lorsque Cavalcanti en vient, au chapitre 6, à parler de ce qu'éprouvent les amants, il s'attache aux formosi que l'éclair de la divinité qui brille en eux rend semblables aux statues divines, se référant au Phèdre (251a). Puis il commente le discours de Pausanias, lorsque celui-ci distingue la Vénus céleste de la Vénus vulgaire :

Celle-là en effet, par un amour inné, est irrésistiblement portée à comprendre la beauté de Dieu < dei pulchritudinem >, celle-ci, grâce à l'amour qui est le sien, à procréer cette beauté dans les corps. Celle-là accueille d'abord en soi la fulguration de la divinité, puis elle la transmet à la deuxième Vénus; celle-ci fait passer dans la matière du monde des étincelles de cette fulguration ; c'est par la présence de telles étincelles que chaque corps mondain paraît beau dans la mesure de sa capacité. L'esprit humain perçoit par les yeux la beauté de ces corps ; lui aussi possède deux puissances puisqu'il a la puissance de comprendre et celle d'engendrer: ces deux puissances jumelles sont en nous les deux Vénus, accompagnées aussi de deux Amours jumeaux. Dès que la beauté d'un corps humain s'offre à nos yeux, notre intelligence, qui est en nous la première Vénus, la vénère et la chérit comme une image de la beauté divine < diuini decoris imaginem > et par elle est entraînée fréquemment vers cette Beauté. De son côté la puissance d'engendrer, notre deuxième Vénus, désire engendrer une beauté semblable $<$ formam similem $\rangle$. Des deux côtés il y a donc Amour. Là désir de contempler, ici désir d'engendrer la beauté < hic generande pulchritudinis desiderium >. Et chacun des deux Amours est honnête et louable, car tous deux s'attachent à l'image divine. (II.7)

Il ne s'agit pas de préférer l'amour de la beauté du corps (formam corporis) à l'amour de la beauté de l'âme (pulchritudini animi), il s'agit plutôt de reconnaître dans la beauté corporelle la beauté supérieure de Dieu: l'amour du corps est essentiellement contemplation, ce qui nous renvoie à l'excellence des amours masculines ;

(...) il y a entre les amants échange de beauté < pulchritudo pro pulchritudine >. L'homme fait jouit par les yeux de la beauté du plus jeune. Le plus jeune atteint par l'esprit la beauté de l'homme fait. Qui n'est beau que de corps < qui solo corpore formosus est > acquiert grâce à ce commerce la beauté de l'âme et qui n'est beau que de l'âme < Qui solo animo est decorus > rassasie de beauté corporelle les yeux du corps. (II.9)

Echange grâce auquel l'acquisition de la beauté de l'âme se révèle plus précieux que celle de la beauté du corps.

Il est à remarquer combien disparaissent les différences de signification entre les adjectifs pulcher, decorus et formosus (voir à ce sujet l'étude de P. Monteil, Beau et laid en latin. Etude de vocabulaire, Paris, 1964, p. 156 : «Ce n'est (...) pas un hasard si presque tous les vocables latins exprimant la beauté présentent (à l'exception de formosus) une acception morale qui aide à mieux interpréter l'acception proprement esthétique. »). Par la conception divine de la Beauté, Ficin fonde une nouvelle alliance entre le corps et l'âme, qui sera amplifiée dans la Théologie platonicienne à propos de forma. C'est ce que nous verrons par la suite.

16 A la fin du discours, nous avons donc progressé dans la compréhension de la Beauté : Dei bonitas > diuina pulchritudo > duae Veneres $>$ pulchritudo animi.

17 Le troisième discours ne parle pas expressément du Beau; le commentaire du discours d'Eryximaque insiste plutôt sur l'universalité de l'Amour, sur le mutuel amour qui 
conserve l'unité des parties (III.2) : cette concordia l'emporte sur la discordia. La dernière phrase réitère la formation de l'homme à la vertu et au bonheur par et dans l'Amour (III.4).

\section{Le commentaire du discours d'Aristophane}

18 Ainsi, quand nous abordons le commentaire du discours d'Aristophane par Landino, une pause a été en quelque sorte ménagée dans le texte. Rien de plus précis, pour le moment, ne semble pouvoir être dit sur la Beauté. Alors même que, chez Platon, le vocabulaire de la beauté - notamment le champ lexical de «kalos »- continuait d'être utilisé par le médecin Eryximaque, chez Ficin rien de tel. Il est alors intéressant de se demander comment et pourquoi Landino utilise à nouveau l'idée de pulchritudo pour interpréter un texte platonicien qui, naguère, n'avait plus recours à la notion de beauté. Ce chassé-croisé sémantique (ne pas recourir au vocabulaire de la beauté, alors que Platon y recourt / recourir au vocabulaire de la beauté, alors que Platon n'y recourt pas) enrichit-il l'interprétation de Platon et des commentaires mêmes de Cavalcanti ?

Landino est d'abord présenté comme homme d'un savoir exceptionnel (uir doctrina excellens) et poète orphique et platonicien (orphicum platonicumque poetam). Quant au discours d'Aristophane, il est dit obscur et compliqué (obscuram et implicatam sententiam) (IV, 1). L'oratio de Landino a pour but de mettre au clair, d'expliciter le sens du texte platonicien par un développement clair (explicare). C'est la première fois que Ficin met en scène un commentaire herméneutique :

Bien que Giovanni Cavalcanti nous ait par son sérieux dispensé des détours d'une longue discussion, l'opinion d'Aristophane, enveloppée en des termes fort obscurs < uerbis obscurissimis >, exige encore quelque élucidation et éclaircissement < enodationem adhuc aliquam lucemque $>$. (IV.1)

Landino commence par exposer le texte de Platon sur la nature primitive de l'homme selon Aristophane. Mais cette anthropologie fantastique doit trouver son véritable exégète en la personne du commentateur ficinien qui définit ainsi sa méthode :

Voilà, parmi tant d'autres, les choses, fantastiques et prodigieuses < monstris portentisque similia >, que conte Aristophane. Mais il y a lieu de penser que sous ces sortes de voiles se cache un divin mystère $<$ sub quibus quasi uelaminibus quibusdam diuina mysteria >. Car c'était la coutume chez les anciens théologiens de celer à l'ombre de figures leurs secrets divins et purs < sacra puraque arcana >, pour les préserver de la souillure du profane impur. Au demeurant nous ne pensons pas que tous les détails évoqués dans les figures qui précèdent et dans d'autres concernent précisément le sens. Selon Augustin lui-même, l'on ne doit pas penser que tout ce qui est figuré dans la figure soit signifiant. Car outre les éléments signifiants, bien des détails s'ajoutent pour les besoins de l'ordre et de la liaison. Seul le soc fend la terre, mais pour que cela soit possible, à ce soc s'unissent les autres membres de la charrue. Voici donc, ramené à l'essentiel, l'objet proposé de notre interprétation. (IV.2)

Pour Landino, il est question de réfléchir sur le sens figuré du discours d'Aristophane, la figura désignant en rhétorique le symbole et l'allégorie qui structurent le plus souvent le discours des poètes, le distinguant du langage quotidien ou de la prose éloquente dont le style doit se caractériser par la simplicité.

Le fondement de l'explication réside dans le fait que, lorsque Aristophane dit «les hommes », il veut dire "les âmes des hommes ». Tout le récit est à comprendre comme 
celui de la chute des âmes qui voulurent s'égaler à Dieu en se suffisant à elles-mêmes; immergées dans les corps, elles ont perdu la lumière divine qui était leur moitié et désirent la recouvrer pour être à nouveau entières et bienheureuses dans la vision de Dieu (IV.2). Nous retrouvons alors la problématique augustinienne du Tout et des parties ainsi que la dévaluation du corps. Cependant, l'amour qui est cette stimulation vers la lumière divine par le désir de comprendre la nature des choses divines (IV.5), passe par la vue de la beauté physique des adolescents et le dernier chapitre, qui évoque la reconduite des âmes au ciel par l'Amour dispensateur de béatitude et de joie éternelle, décrit le banquet céleste où les amants jouissent de la beauté des aimés; jouissance exquise et douce qui n'éprouve aucunement l'envie :

C'est pourquoi, comme Platon le dit dans le Phèdre, l'envie n'existe pas dans le chœur divin. Car, rien n'étant plus agréable que de posséder ce qu'on aime, chacun, jouissant de ce qu'il aime, vit content et comblé. Et si deux amants viennent à posséder l'objet de leurs délices, chacun d'eux se reposera dans la possession de l'être aimé et n'aura nul souci de savoir si l'autre possède un bien-aimé plus beau < neque curam quidem ullam habebit, num alter pulchriori potiatur amato >. (IV.6)

Au-delà de l'inuidia, la beauté apparait comme un bien absolu. Landino accorde les deux définitions, métaphysique (splendeur) et phénoménologique (harmonie des proportions), de la beauté des corps des amants afin de construire son commentaire; le vocabulaire de la beauté (pulchritudo) ne saurait être absent d'une interprétation qui n'envisage pas, depuis le premier commentaire de Cavalcanti, de parler de l'Amour sans parler du Divin ni de parler du Divin sans parler du Beau. Alors la fable d'Aristophane prend une signification sublime : Landino finit par nous transporter à la table de Dieu (ad celestem mensam), ce que le Banquet de Platon n'avait jamais osé faire. Alors que le banquet du philosophe se distingue par la mise en question de la connaissance (scientia) et s'efforce de définir l'Amour, Landino place l'amour de Dieu (caritas) au-dessus de ce souci de connaissance, parce que, précisément, la connaissance véritable de Dieu est impossible et que Dieu veut être aimé. La pulchritudo estce qui révèle dans toute sa clarté la prééminence de l'amour divin dans la relation à l'aimé :

«Nous-mêmes nous entourons de notre affection non pas ceux qui nous connaissent, mais ceux qui nous aiment: car nous tenons pour ennemis bien des gens qui nous connaissent. Donc ce qui nous ramène au ciel, ce n'est point la connaissance de Dieu, mais l'amour. ».

24 Ainsi interprété, le discours d'Aristophane devient l'occasion de méditer sur cette nouvelle connexion : Amor > pulchritudo > caritas / scientia.

Ficin, mettant en scène Landino, met en scène la poésie et lui assure une compétence certaine pour représenter les conceptions platoniciennes majeures.

\section{Conclusion}

Le personnage mis en scène par Haruki Murakami, dont nous avons cité les propos en exergue, est bibliothécaire; il s'adresse à Kafka, pseudonyme d'un jeune garçon qui s'est enfui de sa maison de Tokyo. Se nommant Oshima, il représente à sa manière le commentaire incarné pour le XXIème siècle du discours d'Aristophane dont il rappelle l'existence à l'adolescent. Il se définit lui-même comme « un absurde hermaphrodite gay et abîmé » (H. Murakami, p. 279) et décrit ainsi sa différence (p. 244 et p. 246 dont nous citons l'extrait) : 
du discours d'Aristophane que la tristesse évidente d'oshima, " hermaphrodite homosexuel » (Murakami 2008 : 403) qui songe à son partenaire, partie manquante de luimême, spécialement « à la saison de la lune pâle, ou quand les oiseaux s'envolent vers le sud... (...) C'est comme si on entrait à nouveau dans une chambre pleine de nostalgie qu'on a quittée il y a longtemps. » (Murakami 2008 : 404). Ficin, en déplaçant du domaine mythologique et moral au domaine métaphysique le propos d'Aristophane, non seulement pratique une méthode de lecture caractéristique de la tradition néoplatonicienne -il écrit dans la Summa dialogi placée en tête de sa traduction du Conuiuium: haec disputatio tota fere est allegorica et metaphysica-, mais surtout nous fait entrer dans une philosophie de la joie, dans ce platonisme heureux dont avait parlé Apulée (De Magia, 64.3 ) : Ceterum Platonica familia nihil nouimus nisi festum et laetum et sollemne et superum et caeleste.

\section{BIBLIOGRAPHIE}

CASSIRER E. 1980. M. Ficinos Theorie des Schönen im Kontext des Platonismus, Heidelberg.

COULTER J. 1976. The Literary Microcosm. Theories of Interpretation of the Later Neoplatonists, Leiden Brill.

FONTANIER J.-M. 2008. La Beauté selon saint Augustin, Presses Universitaires de Rennes, 2 ème édition augmentée.

LAURENS P. 2002. «Platonis aperiamus sententiam : la Lectura Platonis dans le Commentaire sur le Banquet », in : Les mystères Platoniciens, Actes du XLIIe Colloque International d'Etudes Humanistes. Centre d'Etudes supérieures de la Renaissance. Tours, 7-10 juillet 1999, p. 17-28. 
LAURENS P. 2002. Marsile Ficin, Commentaire sur Le Banquet de Platon, De l'amour, Texte et traduction, Les Belles Lettres, Paris, 2002. [NB : L'ouvrage comprend une vaste bibliographie].

MAGNARD P. 2001. Les Platonismes : Marsile Ficin. Les Platonismes à la Renaissance, Actes du Colloque International tenu en mai 1999 à Paris, Paris.

MARCEL R. 1958. Marsile Ficin (1433-1499), Paris.

MARCEL R. 1964-1970. Marsile Ficin, Théologie platonicienne de l'immortalité des âmes, éd. et trad., Paris (3 vol.).

MONTEIL P. 1964. Beau et laid en latin. Etude de vocabulaire, Paris.

MURAKAMI H. 2006. Kafka sur le rivage, 10/18, « Domaine étranger », Belfond, Paris.

ROBIN L. 1930. Platon, Le Banquet, L. Robin (ed. trad. com.), Paris : Les Belles Lettres.

ROCKE M. 1996. Forbidden Friendships : Homosexuality and Male Culture in Renaissance Florence, Oxford University Press.

TOUSSAINT S. (ed.). 2002 Les mystères Platoniciens : Marsile Ficin ou les mystères Platoniciens, Actes du Colloque International d'Etudes Humanistes, CESR Tours, Cahiers de l'Humanisme, Série $n^{\circ} 2$, Paris.

\section{RÉSUMÉS}

Nous nous proposons d'étudier comment le commentaire du discours d'Aristophane, tel que le compose Marsile Ficin dans son De amore, IV à partir du Banquet de Platon, énonce une spiritualité amoureuse de la beauté humaine en Dieu. Se référant à la beauté, idée platonicienne, absente du discours d'Aristophane, afin de mettre en valeur l'amour de l'amant pour l'aimé, Ficin d'une manière paradoxale christianise Platon.

We shall study how the commentary of the discourse of Aristophanes, written by Marsile Ficin in De amore, IV, inspired by the Banquet of Platon, expresses a spirituality of love of human beauty in God. Referring to Beauty, Platonist Idea, absent from the discourse of Aristophanes, in order to highlight the love of the lover for the loved, Ficin paradoxically christianizes Plato.

\section{INDEX}

Mots-clés : amour, beauté, Dieu

Keywords : beauty, God, Love

\section{AUTEUR}

\section{EVRARD DELBEY}

Professeur de langue et littérature latines à l'Université de Nice. Centre de recherches EA 4081 (Paris IV), « Rome et ses renaissances ». Auteur de plusieurs ouvrages, dont Poétique de l'élégie romaine. Les âges cicéronien et augustéen, Les Belles Lettres, Paris, 2001 et Venance Fortunat ou l'enchantement du monde, Collection «Interférences », Presses Universitaires de Rennes, 2009. 